INSTRUMENTATION \& TECHNIQUES

\title{
An inexpensive multiplexing system for recording multiple physiological responses from several subjects concurrently
}

\author{
D. H. VANDERCAR, H. F. LAMPHEAR, and HERB KARL \\ University of South Florida, Tampa, Florida 33620
}

A complete multiplexing system capable of transducing and recording four physiological responses concurrently from six subjects at a time is described. Skin conductance level was recorded from the nonpreferred hand with the aid of a simple constant-voltage circuit. Temperature was recorded from the left and right middle fingers with thermistors. Integrated electromyographic activity was recorded from the frontalis muscle with a previously described biofeedback circuit. An electromechanical rotary switch serves as a multiplexer. Responses are converted to frequencies by voltage-to-frequency integrated circuits and recorded on a fourchannel AM tape recorder. The records are subsequently transcribed by a Data General computer and InterACT circuitry programmed to count and print frequencies. Data obtained from 36 subjects as they viewed a feature-length film containing explicit violence are presented.

There are instances in which it is necessary and desirable to obtain multiple measurements from a number of subjects concurrently. This was the case in a study we conducted that sought to determine the relationship between subjects' verbal reports and physiological responses to a film containing explicit scenes of violence. This article describes a reliable inexpensive multiplexing system for transducing and recording four physiological responses concurrently from six subjects at a time. In addition, the data obtained from these subjects are presented.

\section{METHOD}

Four physiological responses were recorded from six subjects simultaneously while they were viewing the feature film "Straw Dogs." Subjects were placed in alternate seats in a row that was approximately $10 \mathrm{~m}$ from a $3 \times 3 \mathrm{~m}$ screen. Subjects were connected via leads to a sound-attenuated enclosure that contained the multiplexer, transductive circuitry, and a fourchannel AM tape recorder.

The responses recorded were: temperature from the left and right middle fingers, skin conductance level (SCL) from the nonpreferred first and third fingers, and electromyographic activity (EMG) from the frontalis muscle of the forehead. EMG and SCL electrodes were nonpolarizing silver/silver-chloride units attached to subjects with sticky collars and K-Y jelly. Temperature was recorded with thermistors ${ }^{1}$ attached with Band-Aids and 4-mm-thick foam rubber in a manner that reduced the effects of ambient temperature.

Figure 1 shows the major components of the system. Shielded phonograph wires connect subjects to transductive circuitry and a stepper ${ }^{2}$ that functions as the multiplexer. Once every $3 \mathrm{sec}$, the stepper is advanced by a $28 \mathrm{~V}$ pulse from a BRS-Foringer recycling timer (T1-903) and pulse former (PF-901). This permits the four responses to be sequentially measured from each subject once every $21 \mathrm{sec}$. The stepper advances to a seventh position, prior to being reset, to mark the end of a sequence.

The temperature transducing thermistors are connected via the stepper to a voltage-to-frequency (V-F) converter (Analog Devices AD537j). This integrated circuit converts changes in the thermistor's resistance to 9-V square-wave pulses. The frequency is determined by the resistance of the thermistor and the value of the capacitor shown in Figure 1. Frequency can be computed by the formula $F$ out $=10^{5} /(R \times C)$, where $R$ is the resistance of the termistor at a given temperature and $C$ is the value of the capacitor in microfarads. For Temperature 1 (Figure 1) a skin temperature of $32^{\circ} \mathrm{C}$ will result in a frequency of $182 \mathrm{~Hz}$. Over the range of temperatures encountered, a change of $1^{\circ} \mathrm{C}$ produces an $8-\mathrm{Hz}$ increase or decrease in the output frequency.

EMG biopotentials are switched through the contacts of the stepper to a differential amplifier and integration circuit. This unit is essentially identical to the EMG biopotential instrument described by Waite (1975). Waite's device produces a de analog output proportional to the EMG biopotential of the subject. The analog signal is developed across an RC integrater with a time constant of approximately $.1 \mathrm{sec}$. The resulting voltages are transformed via a second V-F converter to a frequency proportional to integrated EMG activity. The frequencies range between 10 and $150 \mathrm{~Hz}$, depending upon the level of EMG activity of a particular subject.

In order to measure SCL, it is necessary to place independent voltage-divider circuits between each subject and the stepper. The potential appearing across the subject was set at $.5 \mathrm{~V}$ by the voltage-divider circuit, which consists of a $100-\mathrm{ohm}$ potentiometer and a 1.4-V AA mercury battery (Mallory ZM9 or equivalent). Batteries are changed between each group of subjects to insure constant voltage. (This circuit is a simplified version of the one described by Venables and Christie, 1973.) Variations in the subject's skin conductance result in changes in the current flowing through the 464-ohm resistor (see Figure 1), which in turn produce a change in voltage drop across this resistor proportional to the subject's SCL. This voltage is then converted to a square-wave frequency by the V-F converter. A shift in subject SCL of 1 micromho produces a $35-\mathrm{Hz}$ change 


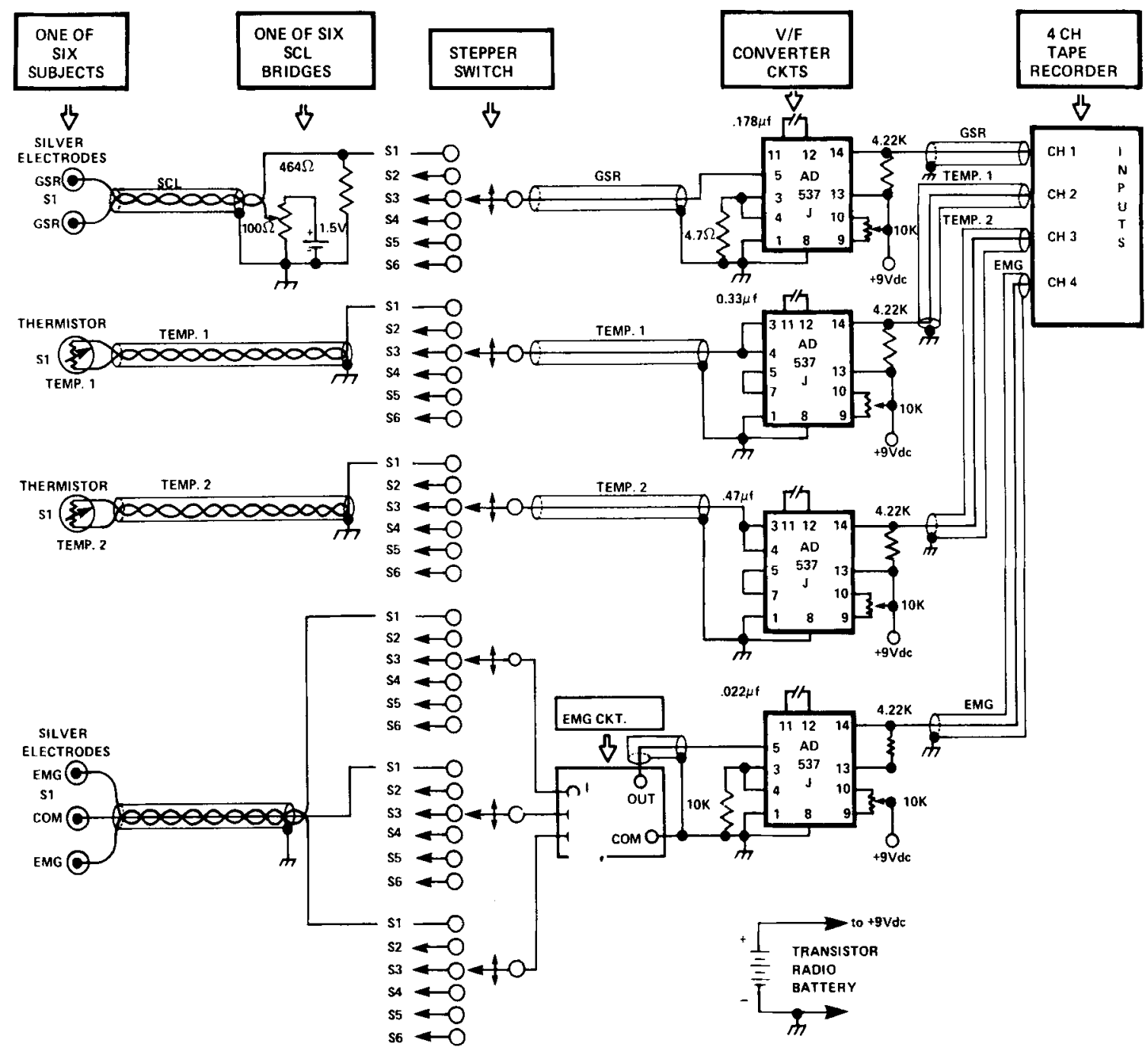

Figure 1. Schematic of the major components of the transductive circuitry, multiplexer, and voltage-to-frequency converters.

in frequency. A 4-micromho SCL equals approximately $108 \mathrm{~Hz}$.

Calibration for the thermistors is accomplished by immersing the units in a water bath of known temperature in the range of the subject's skin temperature. In the case of SCL, precision resistors are substituted for the subjects.

The output of the four V-F converters (Figure 1) is connected to a four-channel quadraphonic AM tape recorder. Frequencies proportional to response magnitude were recorded from each of the six subjects for a duration of $2.5 \mathrm{~h}$. Data from the tapes were subsequently transcribed with the aid of a Data General computer and BRS InterACT interfacing, programmed to count and print frequencies. ${ }^{3}$

This transcription was accomplished by delivering the output of the tape recorder, one channel at a time, into a Schmitt trigger designed to transform the output frequency of the tape recorder into $28-\mathrm{V}$ square-wave pulses. This signal was then fed into one of the inputs of the In terACT interfacing panel.

Software is programmed to count and print out the frequency present during each subject-sample period. A quiet period, occurring when the stepper is in its seventh blank position, signals the beginning of a sequence. Programmed intervals allow the first $1.5 \mathrm{sec}$ of each subject's frequencyencoded response to be ignored prior to sampling. In this fashion, transductive circuitry and switching transients are allowed to stabilize prior to the capture of data. Monitoring of input signals confirmed that the data printed out reliably corresponded to the appropriate subject.

The cost of this multiplexing and transductive system depends to a large extent upon the number of channels and the type of electrodes used. In our case, by resurfacing disposable electrodes, ${ }^{4}$ we were able to construct the system for less than $\$ 300$.

\section{SUPPORTING DATA}

Utilizing this equipment, we examined physiological patterns of arousal produced by viewing the movie "Straw Dogs." This film was chosen for its suspense and numerous scenes of explicit violence. Figure 2 presents the average SCL, hand temperatures, and integrated EMG obtained from 36 subjects who watched the film. Separate analyses of variance revealed a significant increase in SCL $[\mathrm{F}(49,784)=3.96, \mathrm{p}<.001]$, a decrease in both left-hand temperature $[F(49,1323)=3.96$, 


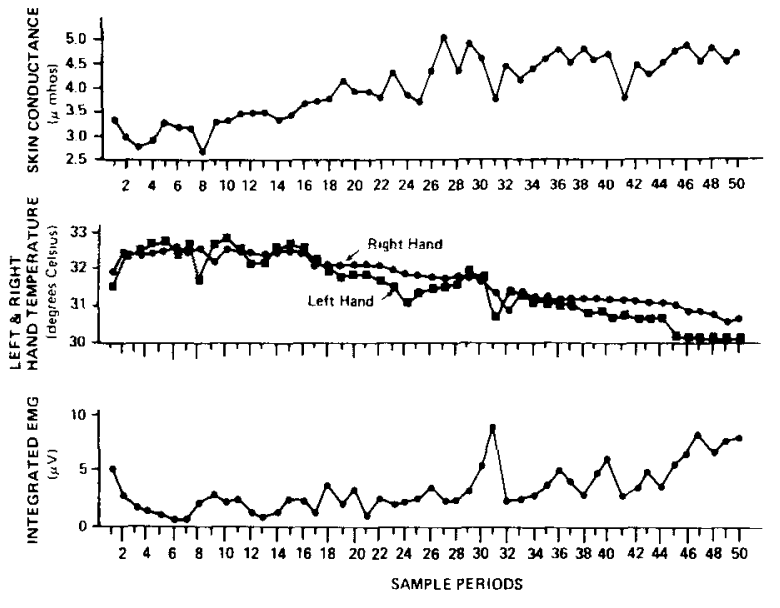

Figure 2. The average skin conductance and temperature of the left and right hands, and EMG activity of all subjects taken during $\mathbf{5 0}$ sample periods.

$\mathrm{p}<.001]$ and right-hand temperature $[\mathrm{F}(49,1323)=$ $5.63, \mathrm{p}<.001]$, as well as an increase in EMG activity of the frontalis muscle of the forehead $[F(49,1176)=$ $1.59, \mathrm{p}<.01]$. Although these changes generally parallel increases in the suspense and violence seen in the film, attempts to identify response changes with specific scenes were not possible. This was in part due to the high density and overlapping nature of the explicit violence.

\section{DISCUSSION}

This system has several distinctive features worth noting. First, it employs a simple yet accurate constantvoltage circuit to transduce SCL. Second, the electromechanical stepper functions as a reliable multiplexer. Finally, the use of inexpensive V-F integrated circuits permits the accurate recording of analog signals with any standard AM tape recorder.

The principal disadvantage of the system involves the length of time between samplings for a given subject. This could be overcome by using recently available solid state CMOS multiplexing circuitry together with analog-to-digital conversion and a digital tape recorder. However, the simplicity of our system has definite advantages for investigators unfamiliar with sophisticated technology and with limited budgets.

\section{REFERENCES}

Venables, P. H., \& Christie, M. J. Mechanisms, instrumentation, recording techniques, and quantification of response. In W. F. Prokasy \& D. G. Raskin (Eds.), Electrodermal activity in psychological research. New York: Academic Press, 1973.

Watte, M. Build a muscle feedback monitor. Popular Electronics, May 1975, 39-42.

\section{NOTES}

1. Yellow Springs Instrument Company Precision Thermistor YSI 44004. Interchangeability is $\pm .2^{\circ} \mathrm{C}$. Units are priced at approximately $\$ 5$.

2. The stepper can be any electromechanical stepping switch with at least six wiper contacts and seven positions. Steppers may be obtained from a variety of surplus supply houses. For best results, the contracts should be electroplated with gold to insure minimal contact resistance.

3. A copy of the program will be made available to interested readers upon request.

4. Pediatric disposable electrodes (DME 95-40) were donated by $\mathrm{K} \& \mathrm{G}$ Healthcare Industries, Inc., P.O. Drawer 168, Goldsboro, North Carolina 27530.

(Received for publication May 2, 1979; revision accepted June 26, 1979.) 